\title{
Bull's-Eye Antenna with Circular Polarization at Millimeter Waves based on Ridge Gap Waveguide Technology
}

\author{
Dayan Pérez-Quintana, Iñigo Ederra and Miguel Beruete
}

\begin{abstract}
In this paper, a Bull's-Eye (BE) antenna with circular polarization (CP) based on ridge gap waveguide (RGW) technology is numerically and experimentally demonstrated. The structure is excited from the bottom with a standard WR-15 waveguide (V-band) coupled through a step transition to a ridge-line that ends in two orthogonal arms of different lengths to generate $\mathrm{CP}$. Radiation is coupled to the top plate by a central diamond slot surrounded by the BE structure, which consists of four concentric periodic corrugations around the slot. Simulations and experimental results are in good agreement, with axial ratio below $1 \mathrm{~dB}$ at the operation frequency and peak gain of $18.4 \mathrm{~dB}$. The antenna has righthanded CP (RHCP) with polarization discrimination of more than $30 \mathrm{~dB}$.
\end{abstract}

Index Terms - Bull's-eye (BE) antenna, circular polarization (CP), ridge gap waveguide ( $R G W$ ) technology, millimeter waves.

\section{INTRODUCTION}

Bull's-Eye (BE) antennas [1] are a part of the larger family of periodic leaky wave antennas (LWA) [2] and in their most typical geometry consist of a metallic plate with a central slot surrounded by concentric corrugations. An interesting characteristic of BE antennas is that they usually radiate at broadside with a relatively high gain, overcoming one of the classical limitations of LWA which is the openstop band effect that precludes radiation at broadside [3]. This is achieved by designing the periodic corrugations in such a way that each half of the BE antenna radiates at opposite directions but in angles very close to broadside, giving rise to a single lobe by merging the radiation of both halves. Another variant of LWAs are the radial line slot antennas and modulated metasurface antennas [4]-[7], which enable the transformation of surface waves into a leaky wave (LW), leading to an enhanced directivity. Since their first conception more than a decade ago [1], [8], BE antennas have been considered as a simple alternative to horns and are now recognized as a separate member of the LWA family, see a historical review in a recent book chapter [9].

At microwave frequencies, BE antennas based on conventional printed circuit board technology perform satisfactorily and admit different implementations of the primary source, like a surface-wave launcher [8] or a simple monopole [10], reducing manufacturing cost and complexity. Nevertheless, as the operation frequency is increased towards millimeter-waves, fully metallic designs are preferred to avoid dielectric losses and $\mathrm{BE}$ antennas follow the classical implementation of a periodic ring structure around a central slot fed by a standard

This work was supported by the Ministerio de Ciencia, Innovación y Universidades under Grants RTI2018-094475-B-I00 (MCIU/AEI/FEDER, UE), TEC2016-76997-C3-1-R. and PID2019-109984RB-C43 (Corresponding author: Miguel Beruete)

D. Pérez-Quintana, is with the Antennas Group, Universidad Pública de Navarra, 31006 Pamplona, Spain, and also with the Institute of Smart Cities (ISC), Universidad Pública de Navarra, 31006 Pamplona, Spain (e-mail: dayan.perez@unavarra.es). waveguide attached to the back plate [1]. In [11] a high gain BE antenna was designed following this standard procedure, using an aluminum plate carved with a sinusoidal profile working at W-band $(77 \mathrm{GHz})$. A large number of corrugations was used to achieve a high gain of about $28.9 \mathrm{~dB}$. This required a large physical area, giving as a result a poor aperture efficiency. In general, it can be said that in BE antennas an increase of the gain implies a reduction of the aperture efficiency, being one of the main limitations of this type of antennas. This was amended in [12] by optimizing the resonant modes inside the grooves in such a way that a gain around $20 \mathrm{~dB}$ was obtained with only two corrugations around the central slot, increasing the aperture efficiency to $32 \%$ in a prototype operating at $60 \mathrm{GHz}$.

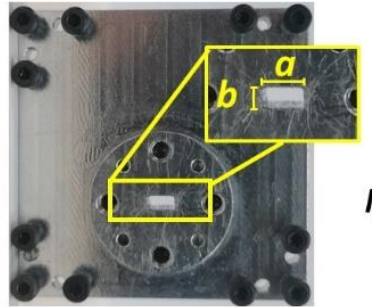

(a)

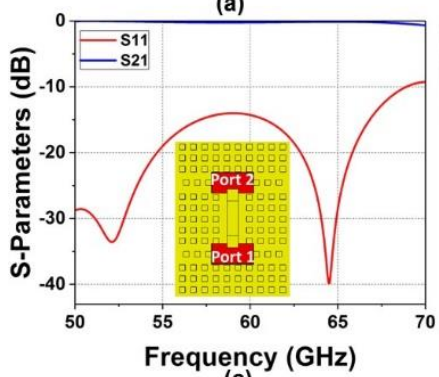

(c)

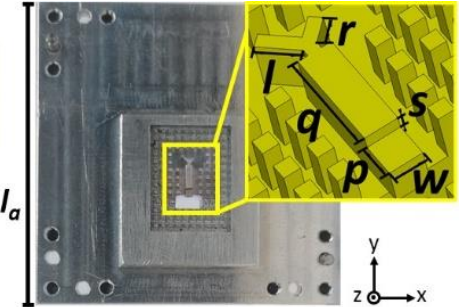

(b)

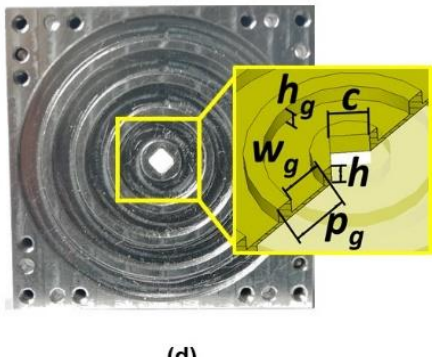

(d)
Fig 1. Photographs and schematics showing the fabricated BE antenna. (a) Bottom view of WR-15 waveguide connection. (b) Feeding system. (c) Simulated reflection and transmission coefficients of the feeding system using a back-to-back evaluation. (d) Top view of concentric periodic corrugations around the diamond-shaped slot. (Insets) Detailed views of each case.

As an alternative to the stringent high accuracy and precision manufacturing constraints required by the previous antennas, a cheaper variant was proposed in [13] by using a low-cost 3-D printing stereolithography technique for a $\mathrm{BE}$ antenna operating at $96 \mathrm{GHz}$ and with tilted radiation with respect to broadside. As noted there, the manufacturing tolerance was at its resolution limits and, consequently,

I. Ederra is with the Antennas Group, Universidad Pública de Navarra, 31006 Pamplona, Spain, and also with the Institute of Smart Cities (ISC), Universidad Pública de Navarra, 31006 Pamplona, Spain (e-mail: inigo.ederra@unavarra.es).

M. Beruete is with the Antennas Group-TERALAB, Universidad Pública de Navarra, 31006 Pamplona, Spain, and also with the Institute of Smart Cities (ISC), Universidad Pública de Navarra, 31006 Pamplona, Spain (e-mail: miguel.beruete@unavarra.es). 
the performance was worse, compared to antennas manufactured using high-accuracy milling techniques. Even with those limitations, a significant gain around $17 \mathrm{~dB}$ was experimentally demonstrated.

Gap waveguide (GW) technology is nowadays under intense research as an alternative to standard waveguides in high-frequency applications [14]-[17]. Amongst the most important characteristics of GW technology is that it does not need electric contact between the bottom and top plates and tolerances are coarser, keeping at the same time a fully metallic structure to avoid dielectric loss. Furthermore, GW technology is naturally adapted to low-profile planar structures, fitting perfectly with the design guidelines of $\mathrm{BE}$ antennas. In this sense, GW can be seen as a trade-off solution between additive manufacturing and high-accuracy milling techniques, encompassing at the same time cost-effective fabrication and good electromagnetic performance.

Hence, the first objective of this manuscript is to implement and demonstrate a $\mathrm{BE}$ antenna using $\mathrm{GW}$ technology to reduce manufacturing complexity compared to standard waveguides and improve the performance compared to prototypes made by additive manufacturing. In this way, the technological reach of GW can be extended towards high-gain, fully planar and fully metallic antennas.

Beyond that, it is evident that the BE geometry is compatible with circular polarization (CP), as the grooves usually have revolution symmetry. However, most BE antennas reported to date have linear polarization, due to the limitation imposed by the primary source, which is usually a rectangular central slot. The first steps towards $\mathrm{CP}$ $\mathrm{BE}$ antennas have already been taken in [18] by using a central crossshaped slot with orthogonal arms of the same length. Although this design can effectively couple a CP wave, it cannot generate CP by itself. Rather, a pair of orthogonal modes in quadrature must be previously generated (for instance, with an orthomode transducer) and coupled afterwards to the cross-shaped slot to radiate a CP wave.

In a recent paper, we demonstrated a diamond slot antenna able to intrinsically produce CP using GW technology [19]. The mechanism to generate CP was simple and effective, based on two orthogonal feeder arms of different lengths placed below the diamond-shaped slot. It was also demonstrated in that paper that the gain could be increased by incorporating a horn taper and a circular groove around the central slot, reaching a value of $11.12 \mathrm{~dB}$ at $67.3 \mathrm{GHz}$. However, even in the optimal case, a significant fraction of the top plate area contributed little to the overall antenna gain, as most of the effective radiation was limited to the surroundings of the slot.

Hence, the second objective of this manuscript is to demonstrate a high-gain antenna with CP by combining the ideas of the diamond slot antenna of [19] with a BE geometry covering maximally the top metallic plane. In this way, we merge together three important concepts: generating CP in a simple way, using GW technology and demonstrating a high-gain in a single antenna without the need of using antenna arrays, which require a complex feeding network [20], [21].

Specifically, in this paper, we design and analyze experimentally a $\mathrm{BE}$ antenna with $\mathrm{CP}$ in ridge GW (RGW) technology. The structure is fed from the bottom using a WR-15 waveguide to RGW transition (note that this is required by the current experimental setups based on standard waveguides and could be removed in future systems operating entirely in $\mathrm{GW}$ technology). $\mathrm{CP}$ is generated by two orthogonal arms with different lengths that excite a top diamondshaped slot, although there are some subtle differences with our previous work [19], as explained in Section II below. The top plate contains four concentric periodic corrugations around the diamondshaped slot covering maximally the metallic plane. An experimental gain of $18.4 \mathrm{~dB}$ at $58.5 \mathrm{GHz}$ is achieved with an aperture efficiency of $9 \%$. An excellent polarization purity with an axial ratio (AR) below
$1 \mathrm{~dB}$ at the central frequency is achieved. The antenna has righthanded CP (RHCP) with polarization discrimination of more than $30 \mathrm{~dB}$. All experimental results have good agreement with the simulations

TABLE I

DESIGN PARAMETERS

\begin{tabular}{ccc}
\hline \hline \multirow{2}{*}{ Parameter } & Description & Values $\left(\mathrm{mm} / \lambda_{0}{ }^{*}\right)$ \\
\hline$l_{a}$ & Length of antenna & $40.0 / 7.80$ \\
$p$ & Step length & $1.05 / 0.20$ \\
$s$ & Step height & $0.38 / 0.07$ \\
$w$ & Step width & $1.00 / 0.20$ \\
$c$ & Slot length & $2.65 / 0.52$ \\
$h_{g}$ & Corrugation height & $1.28 / 0.25$ \\
$p_{g}$ & Corrugation period & $4.36 / 0.85$ \\
$w_{g}$ & Corrugation width & $3.13 / 0.61$ \\
$b$ & WR-15 height & $1.90 / 0.37$ \\
$a$ & WR-15 width & $3.18 / 0.62$ \\
$r$ & Right arm length & $0.78 / 0.15$ \\
$l$ & Left arm length & $1.28 / 0.25$ \\
$h$ & Top plate height & $1.62 / 0.32$ \\
\hline \hline
\end{tabular}

$* \overline{\lambda_{0}=5.1 \mathrm{~mm} \text { at } 58.5 \mathrm{GHz}}$

The paper is organized as follows. In Section II the design and simulation results of the $\mathrm{CP} \mathrm{BE}$ antenna are presented and discussed. Section III is focused on the experimental characterization of the manufactured antenna. Finally, the main conclusions are summarized in Section IV.

\section{ANTENNA Design}

The Transient Solver of the commercial simulator CST Microwave Studio ${ }^{\circledR}$ was used to design and optimize the BE antenna shown in Fig. 1, where all pieces are made of aluminum modeled as a lossy metal with a conductivity of $3.72 \times 10^{7} \mathrm{~S} / \mathrm{m}$. The antenna was optimized to operate at the V-band. Fig. 1(a) shows the WR-15 waveguide connection from the bottom plate with a slot of dimension $a \times b$, necessary for compatibility with our standard experimental setup. The WR-15 to RGW transition consists of a simple step of height $s$ and length $p$, as shown in the top view of the feeding network in Fig. 1(b), with the dimensions outlined in the inset of Fig. 1(b) and the values specified in Table I. Although this feeder has a narrower AR BW than the one used in [19], we opted for this solution because it is simpler, easier to fabricate and fits well with the requirements of BE antennas, which are usually narrowband. As demonstrated in the back-to-back (i.e. two identical transitions connected back-to-back) simulation results of Fig. 1(c), its performance is satisfactory. Within the considered bandwidth (BW) the transition has an excellent matching with a reflection coefficient below $-10 \mathrm{~dB}$ and extremely small insertion loss. Finally, Fig. 1(d) shows the top plate of the antenna with four concentric periodic corrugations of period $p_{g}$, depth $h_{g}$ and width $w g$ filling maximally the metallic plane around the central diamondshaped slot of side $c$ and a slot cavity height $h$; see details in the inset of Fig. 1(d). The pins dimensions are $0.5 \times 0.5 \times 1.5 \mathrm{~mm}$ and the periodicity is $1 \mathrm{~mm}$, the same as in [19].

As explained in detail in [19] the CP is generated by the length difference between the two orthogonal arms at the end of the ridge, $r$ and $l$. In [19], the difference between the left and right arms was nearly $\lambda_{0} / 4$, giving rise to a phase difference of $90^{\circ}$ and therefore to a $\mathrm{CP}$ at the output. However, in the present case the length difference between both arms is $0.5 \mathrm{~mm}$ which is only $\lambda_{0} / 10 \quad(l=1.28 \mathrm{~mm}$ and $r=0.78 \mathrm{~mm}$ ). To ascertain the reason behind this disagreement, we first note that in the present antenna the height of the upper plate and hence the height of the diamond slot must be increased to carve the periodic grooves, with a non-negligible impact on the final result. To 
analyze it, we did a parametric study varying $h$ while keeping a constant difference between arms of $\lambda_{0} / 4$, checking the influence on the AR (note that, without loss of generality, in this study the grooves were removed for simplicity). It is evident in Fig. 2(a) that for small values of $h(<0.9 \mathrm{~mm})$ the structure behaves satisfactorily with low $\mathrm{AR}(\mathrm{AR}<3 \mathrm{~dB})$ in good agreement with the results of [19], but when $h$ increases beyond $0.9 \mathrm{~mm}$ the AR degrades rapidly. A similar study was done fixing the arms length difference to $\lambda_{0} / 10$; see Fig. 2(b). Now the best AR is achieved with $h=1.5 \mathrm{~mm}\left(0.3 \lambda_{0}\right)$. These results suggest that the slot height has some non-trivial effect on the phase difference achieved between orthogonal linear components. Nevertheless, an indepth study of this phenomenon is beyond the scope of this manuscript.

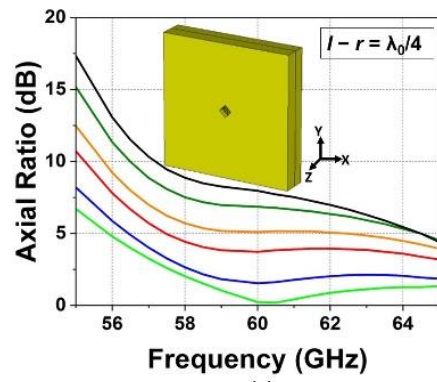

(a)

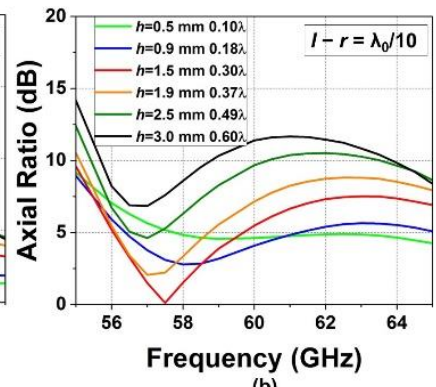

(b)
Fig. 2. Numerical study of the AR magnitude as the height of the top metallic plate $h$ is varied between 0.5 and $3 \mathrm{~mm}$ (corrugations have been removed for simplicity). (a) Arms length difference equal to $\lambda_{0} / 4$. (b) Arms length difference equal to $\lambda_{0} / 10$.

Once the wave is coupled through the slot (of side $c \cong \lambda_{0} / 2$ to guarantee good radiation at the operation frequency), it reaches the top plate and excites the BE structure, as shown in the surface current representation of Fig. 3. The physical mechanism of BE antennas is based on LW excitation and has been extensively described in the past [9]. In the present case, wide grooves were implemented, as they lead to higher gain values as explained in detail in [12], [22]. The grooves support a resonance at the operation frequency (Fig. 3), contributing to the enhancement of gain at broadside. It is also clear that the currents follow a circular shape describing a right-handed CP (RHCP) pattern.

\section{Prototype FABrication AND Result ANAlysis}

The previously discussed BE antenna was manufactured and measured. As RGW technology does not demand high precision manufacturing, standard computer numerical control (CNC) milling machining was selected to manufacture all the pieces. As mentioned above, the material employed was aluminum due to its good conductivity and mechanical properties.

The experimental characterization was done in an anechoic chamber. A PNA network analyzer E8361C (Agilent Technologies) was used to measure the antenna in the frequency range from 55 to $65 \mathrm{GHz}$, with the frequency span discretized in steps of $50 \mathrm{MHz}$. A single port calibration was performed to measure the reflection coefficient of the BE antenna. Fig. 4 shows the main simulation and measurement results. As shown in the solid red curve of Fig. 4(a), good matching is obtained in all the considered BW with $\mathrm{S}_{11}<-10 \mathrm{~dB}$

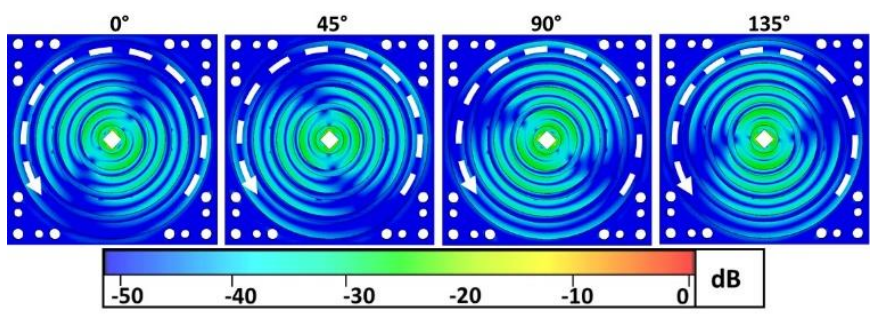

Fig. 3 Upper view of the surface current magnitude on the $\mathrm{BE}$ antenna at $58.5 \mathrm{GHz}$ and four different snapshots.

from 54.3 to $63.7 \mathrm{GHz}$, which represents a fractional BW of $16.2 \%$, in good agreement with the simulation results (dashed red curve).

The gain, AR and radiation patterns of the antenna were measured by following the next procedure. A test horn antenna (Mi-Wave 261) with linear polarization was placed at a distance of $2000 \mathrm{~mm}$ from the $\mathrm{BE}$ antenna (the farfield distance at the operation frequency is $1260 \mathrm{~mm}$ ). In this position, the BE antenna was swept in elevation from $-90^{\circ}$ to $90^{\circ}$ with a step of $0.5^{\circ}$, within the frequency span mentioned above. Two different measurements were taken at each point by rotating the horn antenna (Mi-Wave 261) in two orthogonal positions. This is required because the test antenna has linear polarization and we need both orthogonal components to retrieve the $\mathrm{CP}$ response in the post-processing, which is based on the method described in [23].

The experimental AR is plotted in the solid blue curve of Fig. 4(a). Taking as a criterion that the wave has $\mathrm{CP}$ when the AR is below $3 \mathrm{~dB}$, we find that the CP BW goes from 57.7 to $61.4 \mathrm{GHz}$, which represents a fractional $\mathrm{BW}$ of $6.3 \%$. The minimum value $(0.59 \mathrm{~dB})$ is around 60 GHz. In the simulation, the CP BW goes from 56.7 to $58.8 \mathrm{GHz}$, a fractional $\mathrm{BW}$ of $3.6 \%$, see dashed blue curve in Fig. 4(a), and a minimum AR of $1 \mathrm{~dB}$ is obtained at $58 \mathrm{GHz}$.

The measured gain (Fig. 4(b) red solid curve) was obtained applying the gain transfer (or gain comparison) method particularized to antennas with arbitrary polarization [23], [24]. In fair agreement with the simulation results (red dashed curve) the measured gain (solid red curve) reaches a maximum value of $18.4 \mathrm{~dB}$ at $58.5 \mathrm{GHz}$ (which is the frequency of minimum AR) and decays away from the operation frequency, as typically happens in $\mathrm{BE}$ antennas. The aperture efficiency is approximately $9 \%$, above the typical value of $\mathrm{BE}$ antennas.

Finally, Fig. 4(c) and (d) show the co-polarized and cross-polarized radiation patterns at $58.1 \mathrm{GHz}$, analyzed in two different planes: Fig. 4(c) $\varphi=0^{\circ}$ ( $x-z$ plane); and Fig. 4(d) $\varphi=90^{\circ}$ ( $y-z$ plane). The experimental results show an excellent polarization isolation of more than $30 \mathrm{~dB}$ at broadside, a main lobe of beamwidth equal to $11^{\circ}$ and a small side lobe level of $-9.7 \mathrm{~dB}$ in both cutting planes, in good agreement with the simulation results. These results improve our previous diamond-horn-groove (DHG) antenna discussed in [19] thanks to the addition of the BE structure, which optimizes the radiation on the top plane. 

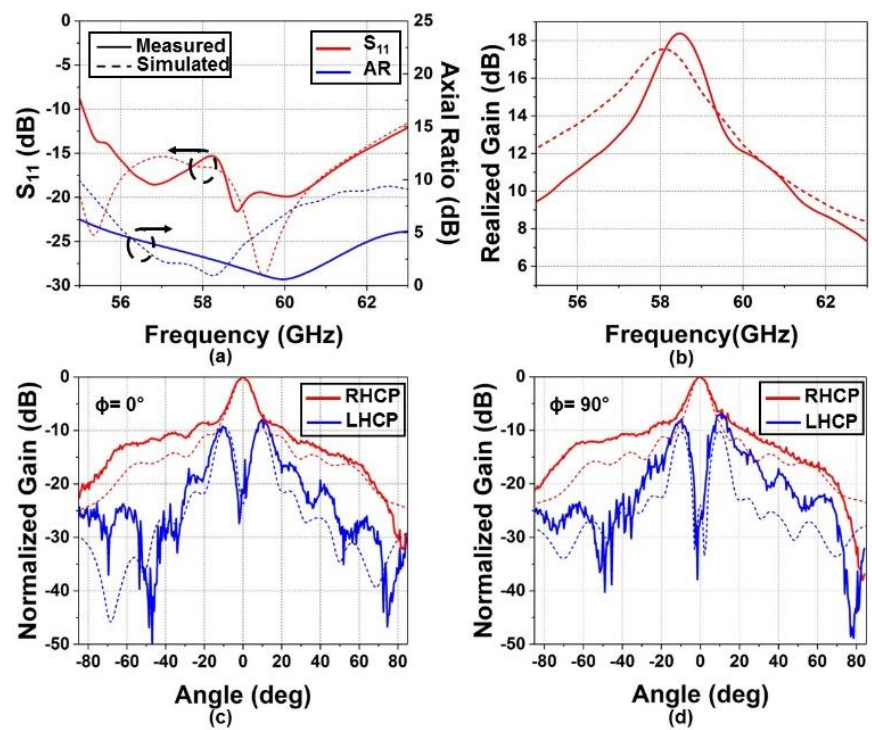

Fig. 4. Simulated (dashed curves) and experimental (solid curves) results of the BE antenna. (a) Reflection coefficient magnitude (red curve) and axial ratio (blue curve). (b) Realized gain at broadside $\left(\varphi=0^{\circ}\right.$ and $\left.\theta=0^{\circ}\right)$. Co-polarized and cross-polarized radiation pattern at $58.1 \mathrm{GHz}$, (c) $\varphi=0^{\circ},(x-z$ plane) (d) $\varphi=90^{\circ},(y-z$ plane $)$. The reference coordinate axes are the same in all figures and are shown in the Fig. 1(b).

TABLE II

COMPARISON BETWEEN DIFFERENT MiLLIMETER-WAVE ANTENNAS

\begin{tabular}{|c|c|c|c|c|c|}
\hline Ref & $\begin{array}{c}\text { Size } \\
(\mathrm{mm} / \lambda)\end{array}$ & $\begin{array}{l}\text { Number } \\
\text { of } \\
\text { Elements }\end{array}$ & $\begin{array}{c}\text { Fractional } \\
\text { BW } \\
\text { (Total BW)* }\end{array}$ & $\begin{array}{c}\text { Fractional } \\
\text { BW } \\
(\text { Total BW) }\end{array}$ & $\begin{array}{c}\text { Peak } \\
\text { Gain- } \\
\left(\begin{array}{c}\text { GBW }) \\
\text { ) }\end{array}\right.\end{array}$ \\
\hline [25] & $\begin{array}{c}80 \times 63 \\
(16 \times 13)\end{array}$ & $16 \times 16$ & $\begin{array}{c}14 \% \\
(57-66)\end{array}$ & $\begin{array}{c}5.0 \% \\
(59-62)\end{array}$ & $\begin{array}{c}32.3 \\
(96.9)\end{array}$ \\
\hline [26] & $\begin{array}{c}2.4 \times 2.4 \\
(0.5 \times 0.5)\end{array}$ & $4 \times 4$ & $\begin{array}{c}14.1 \% \\
(56-65)\end{array}$ & $\begin{array}{l}21.1 \% \\
(55-68)\end{array}$ & $\begin{array}{c}19.5 \\
(253.5)\end{array}$ \\
\hline [27] & $\begin{array}{c}10 \times 15 \\
(2.0 \times 3.0)\end{array}$ & $2 \times 2$ & $\begin{array}{c}6.5 \% \\
(58-62)\end{array}$ & $\begin{array}{c}4.9 \% \\
(59-62)\end{array}$ & $\begin{array}{c}12.5 \\
(37.5)\end{array}$ \\
\hline [28] & $\begin{array}{c}7.9 \times 7.9 \\
(1.6 \times 1.6)\end{array}$ & $2 \times 2$ & $\begin{array}{c}18 \% \\
(56-67)\end{array}$ & $\begin{array}{c}16.7 \% \\
(56-66.2)\end{array}$ & $\begin{array}{c}14.6 \\
(148.9)\end{array}$ \\
\hline [29] & $\begin{array}{c}5.5 \times 3.0 \\
(1.1 \times 0.6)\end{array}$ & $1 \times 1$ & $\begin{array}{c}17.13 \% \\
(58.6-69.7)\end{array}$ & $\begin{array}{c}13.3 \% \\
(57-65)\end{array}$ & $\begin{array}{c}7.4 \\
(59.2)\end{array}$ \\
\hline [19] & $\begin{array}{c}30 \times 30 \\
(6.0 \times 6.0)\end{array}$ & $1 \times 1$ & $\begin{array}{c}14.64 \% \\
(60.3-69.6)\end{array}$ & $\begin{array}{l}17.32 \% \\
(60-71)\end{array}$ & $\begin{array}{c}11.12 \\
(122.3)\end{array}$ \\
\hline $\mathrm{BE}$ & $\begin{array}{c}40 \times 40 \\
(7.8 \times 7.8)\end{array}$ & $1 \times 1$ & $\begin{array}{c}16.16 \% \\
(54.3-63.7)\end{array}$ & $\begin{array}{c}6.3 \% \\
(57.7-61.4)\end{array}$ & $\begin{array}{c}18.4 \\
(68.0)\end{array}$ \\
\hline
\end{tabular}

* Defined as $\mathrm{S} 11<-10 \mathrm{~dB}$. Total $\mathrm{BW}$ is in $\mathrm{GHz}$

${ }^{+}$Defined as $\mathrm{AR}<3 \mathrm{~dB}$. Total $\mathrm{BW}$ is in $\mathrm{GHz}$

\# Peak Gain is in $\mathrm{dB}$. For the gain-bandwidth product (GBW) calculation, the $\mathrm{BW}$ is defined as $\mathrm{AR}<3 \mathrm{~dB}$

Table II presents a comparison between our BE design and other CP antennas recently reported in the literature that operate near $60 \mathrm{GHz}$ [25]-[29]. In all cases, the results are analyzed quantitatively. As can be seen there, our BE antenna has in general good radiation characteristics. It has a moderate gain value $(18.4 \mathrm{~dB})$. Note that this gain is similar to that achieved by antenna arrays, which are comparatively more complex that our $\mathrm{BE}$ antenna based on a single radiating element and a simple feeding network. As it is usual in $\mathrm{BE}$ antennas, the operation bandwidth $(6 \%$; we take here the AR BW as it is the interesting one for applications) is narrow, impacting directly in the gain-bandwidth product (GBW), although there are other examples in the Table in which it is even narrower. Thus, from this comparison, it can be said that the designed $\mathrm{BE}$ antenna can be a good choice for moderate gain and narrowband applications at millimeter-waves.

\section{CONCLUSIONS}

In this work a $\mathrm{BE}$ antenna with $\mathrm{CP}$ operating in the $\mathrm{V}$-band has been numerically and experimentally demonstrated. A feeding system based on RGW technology has been implemented to couple the wave to the radiating diamond-slot. The $\mathrm{CP}$ generation mechanism is based on the length difference between two orthogonal arms at the end of the ridge. However, due to the larger height of the diamond slot, the length difference has to be reduced from $\lambda_{0} / 4$ to nearly $\lambda_{0} / 10$. A BE structure at the output, consisting of four concentric periodic corrugations around the slot contribute to enhance the gain by covering maximally the top metallic plane. The simulated and experimental results are in good agreement, demonstrating good radiation characteristics with a compact and low profile structure. A high gain of around $18.4 \mathrm{~dB}$ at $58.5 \mathrm{GHz}$, with an aperture efficiency of $9 \%$ is obtained. A good CP purity with an AR of $0.59 \mathrm{~dB}$ at $60 \mathrm{GHz}$ is demonstrated. More than $30 \mathrm{~dB}$ of cross-polarization isolation is observed in the measured radiation patters, with a main lobe beamwidth equal to $11^{\circ}$ and small side lobe level of $-9.7 \mathrm{~dB}$. This is the first BE antenna based on RGW technology and able to generate $\mathrm{CP}$ by itself, with excellent performance and relatively cheap manufacturing requirements.

\section{REFERENCES}

M. Beruete et al., "Very low-profile 'Bull's Eye' feeder antenna," IEEE Antennas Wirel. Propag. Lett., vol. 4, no. 1, pp. 365-368, 2005.

[2] A. A. Oliner, "Leaky-Wave Antennas," in Antenna Engineering Handbook, R. C. Johnson, Ed. New York: Mc Graw-Hill, 1993.

[3] M. Guglielmi and D. R. Jackson, "Broadside radiation from periodic leaky-wave antennas," IEEE Trans. Antennas Propag., vol. 41, no. 1, pp. 31-37, 1993

[4] J. L. Gomez-Tornero, A. J. Martinez-Ros, N. Llombart, D. Blanco, and E. Rajo-Iglesias, "Near-field focusing with holographic twodimensional tapered leaky-wave slot antennas," in 2012 6th European Conference on Antennas and Propagation (EUCAP), 2012, pp. 234-238.

[5] G. Minatti et al., "Modulated Metasurface Antennas for Space: Synthesis, Analysis and Realizations," IEEE Trans. Antennas Propag., vol. 63, no. 4, pp. 1288-1300, 2015.

[6] M. Faenzi et al., "Realization and Measurement of Broadside Beam Modulated Metasurface Antennas," IEEE Antennas Wirel. Propag. Lett., vol. 15, pp. 610-613, 2016.

[7] Y. F. Wu and Y. J. Cheng, "Two-Dimensional Near-Field Focusing Folded Reversely Fed Leaky-Wave Antenna Array With High Radiation Efficiency," IEEE Trans. Antennas Propag., vol. 67, no. 7, pp. 4560-4569, Jul. 2019.

[8] P. Baccarelli, P. Burghignoli, G. Lovat, and S. Paulotto, "A novel printed leaky-wave 'bull-eye' antenna with suppressed surface-wave excitation," in IEEE Antennas and Propagation Society Symposium, 2004., 2004, vol. 1, p. 1078-1081 Vol.1.

[9] M. Beruete, U. Beaskoetxea, and T. Akalin, "Flat Corrugated and Bull's-Eye Antennas," in Aperture Antennas for Millimeter and Sub-Millimeter Wave Applications. Signals and Communication Technology, 1st ed., A. Boriskin and R. Sauleau, Eds. Cham, Switzerland: Springer International Publishing, 2018, pp. 111-141.

[10] U. Beaskoetxea, A. E. Torres-Garcia, and M. Beruete, "Ku-Band Low-Profile Asymmetric Bull's-Eye Antenna With Reduced Sidelobes and Monopole Feeding," IEEE Antennas Wirel. Propag. Lett., vol. 17, no. 3, pp. 401-404, Mar. 2018.

[11] U. Beaskoetxea et al., "77-GHz High-Gain Bull's-Eye Antenna With Sinusoidal Profile," IEEE Antennas Wirel. Propag. Lett., vol. 
14, pp. 205-208, 2015.

[12] U. Beaskoetxea and M. Beruete, "High Aperture Efficiency Wide Corrugations Bull's-Eye Antenna Working at $60 \mathrm{GHz}$," IEEE

Trans. Antennas Propag., vol. 65, no. 6, pp. 3226-3230, Jun. 2017.

[13] U. Beaskoetxea, S. Maci, M. Navarro-Cia, and M. Beruete, "3-DPrinted 96 GHz Bull's-Eye Antenna With Off-Axis Beaming," IEEE Trans. Antennas Propag., vol. 65, no. 1, pp. 17-25, Jan. 2017.

[14] P.-S. Kildal, E. Alfonso, A. Valero-Nogueira, and E. Rajo-Iglesias, "Local Metamaterial-Based Waveguides in Gaps Between Parallel Metal Plates," IEEE Antennas Wirel. Propag. Lett., vol. 8, pp. 8487, 2009.

[15] A. Berenguer, V. Fusco, D. E. Zelenchuk, D. Sanchez-Escuderos, M. Baquero-Escudero, and V. E. Boria-Esbert, "Propagation Characteristics of Groove Gap Waveguide Below and Above Cutoff," IEEE Trans. Microw. Theory Tech., vol. 64, no. 1, pp. 27 36, Jan. 2016.

[16] A. U. Zaman and P. Kildal, "Wide-Band Slot Antenna Arrays With Single-Layer Corporate-Feed Network in Ridge Gap Waveguide Technology," IEEE Trans. Antennas Propag., vol. 62, no. 6, pp. 2992-3001, 2014.

[17] J. Liu, A. Vosoogh, A. U. Zaman, and J. Yang, "Design and Fabrication of a High-Gain 60-GHz Cavity-Backed Slot Antenna Array Fed by Inverted Microstrip Gap Waveguide," IEEE Trans. Antennas Propag., vol. 65, no. 4, pp. 2117-2122, Apr. 2017.

[18] S. Alkaraki, Y. Gao, C. Parini, M. Navarro-Cia, and M. Bernete, "Linearly and circularly polarised Bull's eye antenna," 2016 Loughbrgh. Antennas Propag. Conf. LAPC 2016, pp. 14-16, 2017.

[19] D. Perez-Quintana, A. Torres-Garcia, I. Ederra, and M. Beruete, "Compact Groove Diamond Antenna in Gap Waveguide Technology with Broadband Circular Polarization at Millimeter Waves," IEEE Trans. Antennas Propag., pp. 1-1, 2020.

[20] T. Li and F. Fan, "Design of ka-band $2 \times 2$ circular polarization slot antenna array fed by ridge gap waveguide," in 2017 Sixth AsiaPacific Conference on Antennas and Propagation (APCAP), 2017, pp. 1-3.

[21] M. Ferrando-Rocher, A. Valero-Nogueira, J. I. Herranz-Herruzo, and J. Teniente, "60 GHz Single-Layer Slot-Array Antenna Fed by Groove Gap Waveguide," IEEE Antennas Wirel. Propag. Lett., vol. 18, no. 5, pp. 846-850, May 2019.

[22] D. Y. Na, K.-Y. Jung, and Y. B. Park, "Transmission Through an Annular Aperture Surrounded With Corrugations in a PEC Plane," IEEE Antennas Wirel. Propag. Lett., vol. 14, pp. 179-182, 2015.

[23] Bee Yen Toh, R. Cahill, and V. F. Fusco, "Understanding and measuring circular polarization," IEEE Trans. Educ., vol. 46, no. 3, pp. 313-318, Aug. 2003.

[24] G. A. Hurd, "IEEE Standard Test Procedures for Antennas," Electron. Power, vol. 26, no. 9, p. 749, 1980.

[25] M. Ferrando-Rocher, A. Valero-Nogueira, J. I. Herranz-Herruzo, and J. Teniente, " $60 \mathrm{GHz}$ Single-Layer Slot-Array Antenna Fed by Groove Gap Waveguide," IEEE Antennas Wirel. Propag. Lett., vol. 18, no. 5, pp. 846-850, May 2019.

[26] Q. Zhu, K. B. Ng, and C. H. Chan, "Printed circularly polarized open loop antenna array for millimeter-wave applications," in 2017 IEEE International Symposium on Antennas and Propagation \& USNC/URSI National Radio Science Meeting, 2017, vol. 2017Janua, no. 2, pp. 2561-2562.

[27] A. B. Guntupalli and Ke Wu, "60-GHz Circularly Polarized Antenna Array Made in Low-Cost Fabrication Process," IEEE Antennas Wirel. Propag. Lett., vol. 13, pp. 864-867, 2014.

[28] D. J. Bisharat, S. Liao, and Q. Xue, "High Gain and Low Cost Differentially Fed Circularly Polarized Planar Aperture Antenna for Broadband Millimeter-Wave Applications," IEEE Trans. Antennas Propag., vol. 64, no. 1, pp. 33-42, Jan. 2016.

[29] T. H. Jang, Y. H. Han, J. Kim, and C. S. Park, “60 GHz Wideband Low-Profile Circularly Polarized Patch Antenna With an Asymmetric Inset," IEEE Antennas Wirel. Propag. Lett., vol. 19, no. 1, pp. 44-48, Jan. 2020. 